\title{
EVALUATION OF GENETOXIC EFFECT OF RADIATION OVER VEGETAL EMBRYOS IN SPECIES WITH DIFFERENT RADIOSENSIBILITIES
}

\author{
Diana Vrîncianu ${ }^{1}$, Cristina Ionită ${ }^{1}$, Iulia Băra ${ }^{2}$, Dorina Creangă ${ }^{1}$ \\ ${ }^{1}$ Univ. Al. I. Cuza, Faculty of Physics, 11 Blvd. Carol I, 700506, Iasi, România, \\ ${ }^{2}$ Univ. Al. I. Cuza, Faculty of Biology, Iasi, România
}

\section{Article Info}

Received: 22 December 2011

Accepted: 31 January 2012

Keywords: Chromosomal aberration, X-ray, Mitotic index .

\begin{abstract}
This study presents the effects induced by X-rays, medium doses (20-70 Gy), over the meristematic tissues of three cereal species: one of barley and two of wheat. The cytogenetic investigation was based on the response of young seedlings during their very early ontogenetic stages to the ionizing radiation exposure. The study was focused on the identification and counting of the chromosomal aberrations for various doses compared to the control samples. Qualitative evaluation revealed: interchromatidian bridges, expulsed chromosomes or chromosome fragments, and complex aberrations (combination of the already mentioned ones). The different behavior of these three species was discussed over the mitotic index, the number of abnormal ana-telophases and over the percentages of the mitosis phases. In each case, the mitotic index was increased in comparison with the control, and also the number of aberrations was found increased; however no evident mathematical correlations with applied doses could be established.
\end{abstract}

\section{Introduction}

Plants, as an important component of the environmental and ecological systems, need to be taken into account when evaluating the overall fate and exposure at radiations. The biocidal effect of ionizing radiation was revealed soon after it has been discovered. Some other (non-lethal) effects of ionizing radiation on plants include genetic damage, cellular death and genetic mutation. Plant cells can be harmed or killed when they are exposed to large or prolonged time of ionizing radiation. Ionizing radiation exposure can also influence plant growth and development. Most of the important concepts on the spontaneous and induced chromosomal aberrations came from the study of plant tissues, such as root meristems, low number and large-sized chromosomes making some plant species ideal for cytogenetic studies (Natarajan, 2005) [1].The effects induced by the absorption of ionizing radiations in vegetable tissues were studied by different research teams. In some recent 
reports, Wi et al., (2007) [2] observed an inhibitory effect of medium-dose (50 Gy) over the vegetal cells division. Eroglu et al., (2007)[3] studied the mutagenic effects of gamma ray for doses between 50-300 Gy, over Hordeum vulgare (barley) seeds, and observed a decrease of M.I. (mitotic index) at embryonic root tip with increasing doses of gamma radiation (the inhibitory effects of gamma rays on the mitotic index indicated that gamma rays have mutagenic effects on embryonic roots). Cheng et al. (2010) [4] have irradiated Triticum aestivum (wheat) with doses of 10-80 Gy and observed a stimulatory effect over the cells division rate, but also a stimulatory effect over the number of cells with chromosomal aberrations. Since 1995 Okamoto and Tatara [5] have showed that cell division frequency in T. aestivum was decreased by low-dose ionizing radiation; recently Singh and Balyan (2009) [6] also confirmed the genotoxic effects of ionizing radiation, in T. aestivum. In the next we discuss the results of our experiments, focused on the evidence of X-Ray genotoxic effect in two cereal species, barley and wheat irradiated with doses of 22.4 and $67.2 \mathrm{~Gy}$.

\section{Method and samples}

Exposure equipment for ionizing radiation was based on a particle linear accelerator type VARIAN CLINAC 2100SC, which produce 6MV photon beam. The samples were irradiated with doses of $22.4 \mathrm{~Gy}$ and $67.2 \mathrm{~Gy}$, corresponding to 10 and 30 minutes respectively. The time of exposure and doses were calculated at the level of the seeds, using classical computer software of radiotherapy treatment planning system.

The biological material has consisted in caryopses of wheat (one variety) and barley (two varieties: Madalin and Regal). The seeds were all chosen from the same experimental plant population, for each type, in order to diminish the genetic variation. For each sample 30 seeds were chosen, left to soak for 4 hours and to germinate on watered paper support in glass dishes, at controlled environmental parameters (in darkness at $20 \pm 0.5{ }^{\circ} \mathrm{C}$ ), into an INCUCELL thermostatic room, for 24 hours. For all the three types of plants control dishes were arranged, which were not exposed at radiations but kept in the same environmental conditions. After the exposure to X-ray and roots development during 24-48 hours inside the INCUCELL room, root meristeme aliquots were harvested and treated in ethylic alcohol: acid acetic (3:1) for 20 hours (to freeze the cells in their respective stages of mitotic division) being further preserved in $70 \%(\mathrm{v} / \mathrm{v})$ ethylic alcohol.

By applying the Feulgen method [7], the selective coloration of chromosomes for cytogenetic investigation was accomplished. For cytogenetic examination, the microscope slides were prepared through squash method, obtaining single layer cells. The cytogenetic investigation was carried out using an OLYMPUS BX51 microscope with 400x magnitude. 
The microscope survey consisted in qualitative and quantitative analysis, based on counting the cells in every of the four mitosis phase (prophase, metaphase, anaphase, telophase) as well as the in the identification of chromosomal aberrations. There were the mitotic index (M.I.) and aberration index (A.I.) that were calculated with the next formulae:

$$
\begin{aligned}
& M . I .(\%)=\frac{n_{d}}{n_{t}} \\
& \text { A.I. }(\%)=\frac{n_{a}}{n_{t}}
\end{aligned}
$$

$n_{d}$-dividing cells, $n_{t}$ - total number of analyzed cells, $n_{a}$ - number of aberrant divisions. For each experimental variant, five microscope slides were considered with ten observation fields each. In total 12,480 cells were analyzed for Madalin barley, 21,275 cells for Regal barley and 8,910 cells for wheat. Average values and standard deviations (calculated with MsExcell soft) were used for graphical plots. Student $t$-test was applied to assess the statistical significance of the changes in the exposed samples compared to control ones, related to the significance threshold of 0.05 .

\section{Results and discussions}

During the qualitative analysis we observed the presence of the main types of simple and complex chromosomal aberrations for both cereal species (Fig. 1). The photographs were taken with 1,000x microscope magnitude, using immersion oil.

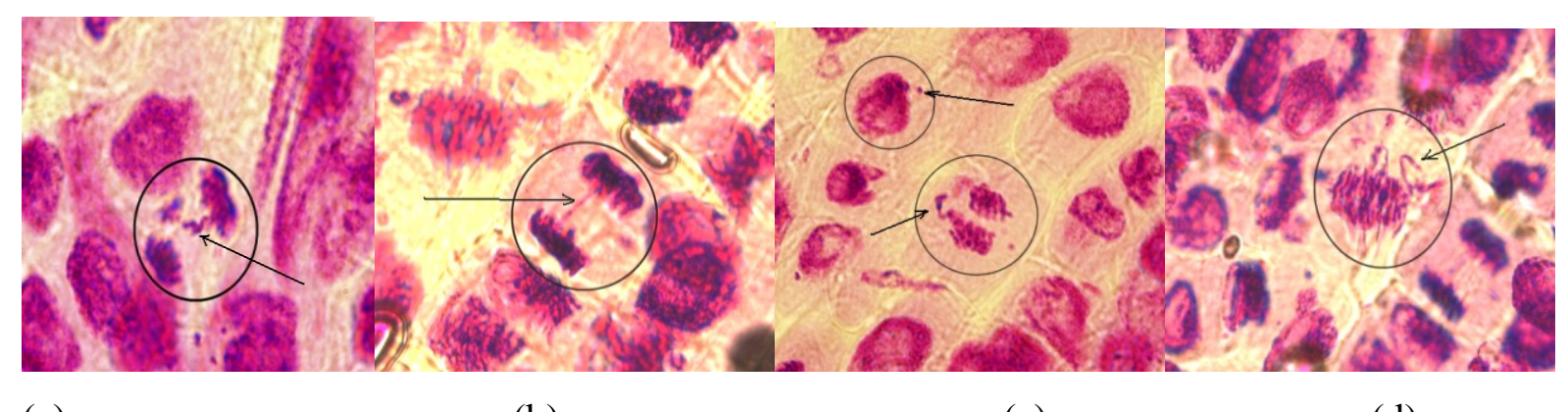

(a)

(b)

(c)

(d)

Fig. 1. (a) Retard chromosomes in Ana-telophase (b) Ana-telophase with interchromatidian bridge (c) Micronucleus (left-up); expelled chromosomes in ana-telophase (middle) (d) Metaphase with expelled chromosomes

Fig. 1 (a) and (c) presents retard and expelled chromosomes in ana-telophase (lagged expelled genetic material between the two division poles respectively expelled genetic material away from the division axis), Fig. 1 (b) shows an interchromatidian bridge in anatelophase (chromosomal aberration consisting in the non separation of the two chromosome arrays between the daughter cell nuclei so that diploid cell resulted with possible modified biological features). Fig. 1 (c) presents a micronucleus (genetic material expelled in a 
previous division and grouped as a micro-nucleus) while Fig. 1 (d) evidences expelled chromosomes in metaphase (the second division phase). The presence of expelled chromosomes in metaphase reveals the early action of ionizing radiations. All the present aberrations confirm the genotoxicity of radiations in cereal species studied.

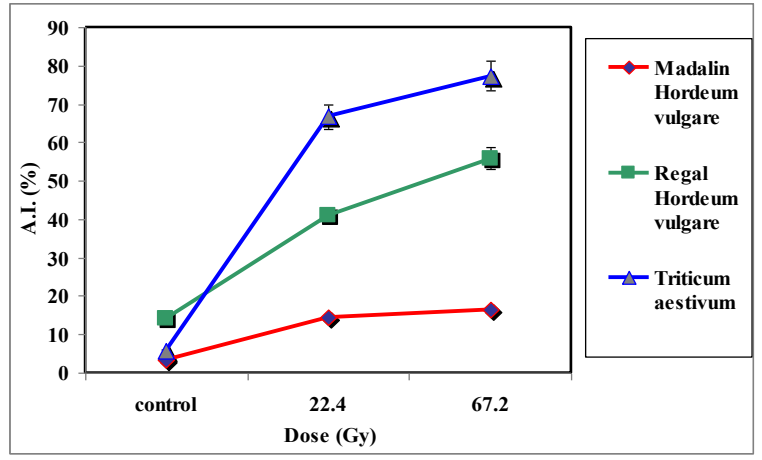

(a)

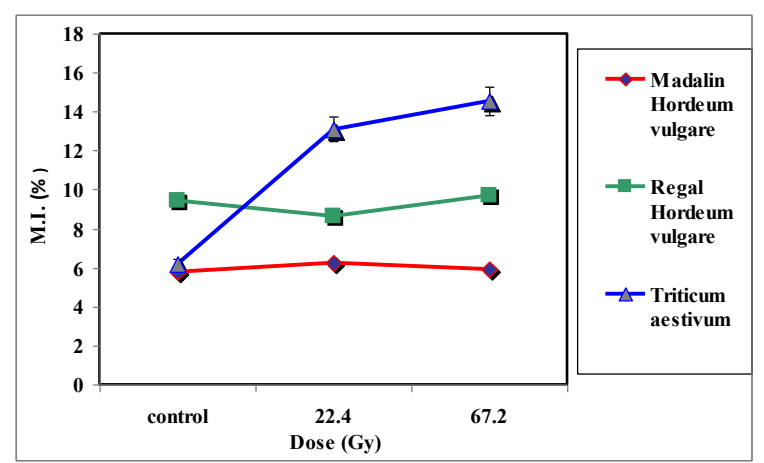

(b)

Fig. 2. a) Mitotic index variation versus radiation; b) Aberration index versus radiation dose

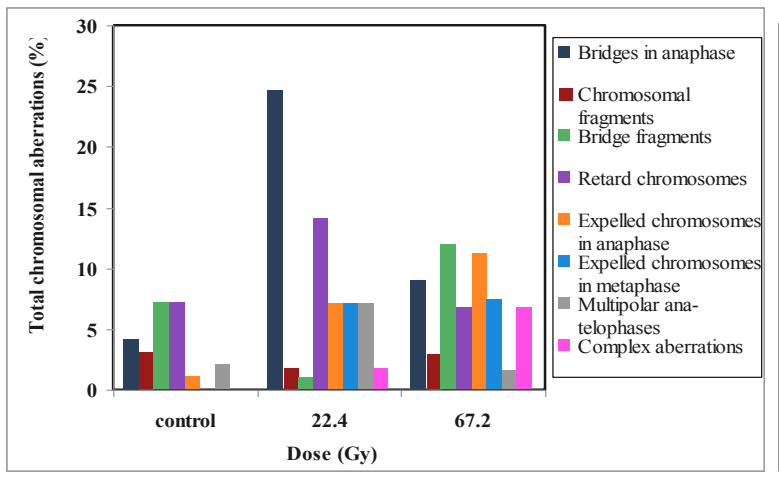

(a)

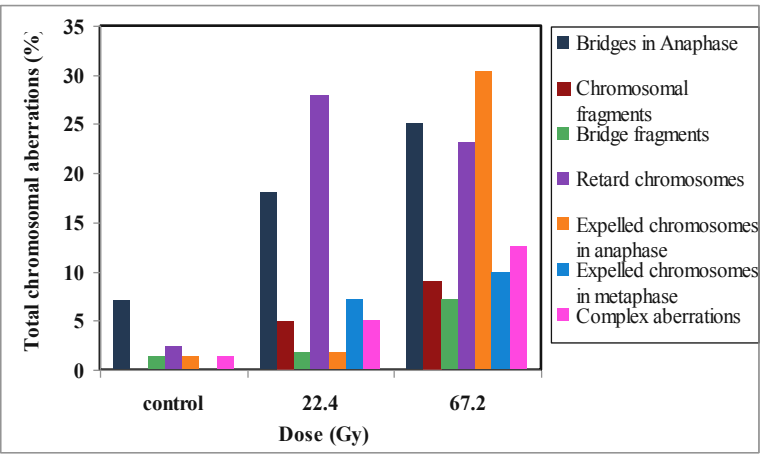

(b)

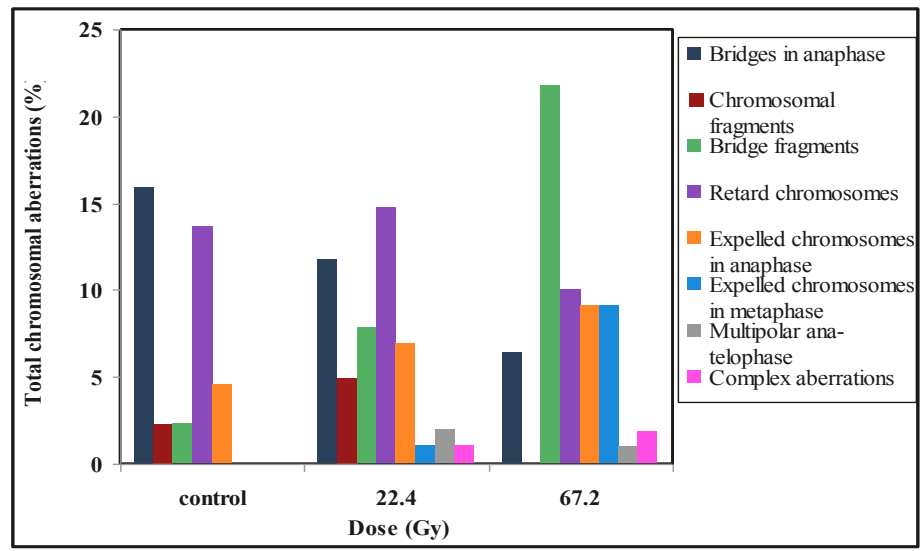

(c)

Fig. 3. Chromosomal aberrations in a) Madalin H. vulgare; b) Regal H. vulgare; c) T. aestivum

Also, the aberration index (Fig. 2. b) was the most increased in irradiated wheat (with $60-70 \%$ in comparison with control) while barley, especially Madalin barley embryos, were evidenced to be most resistant to radiation impact (an increase with $40 \%$ for Regal barley and with10\% for Madalin barley). In Figs. 3, the percentages of all types of chromosomal 
aberrations identified in the analyzed samples are represented. In T. aestivum dose increasing resulted in increased frequency of bridge fragments and expelled chromosomes as well as in diminished frequency of interchromatidian bridges. The rate of complex aberration increased with radiation dose, for all three species studied. The expelled chromosomes in metaphase reveal an early action of ionizing radiation; it can be observed that the increasing of X-rays dose induced dominantly this kind of aberrations. The mitosis phases (prophase, metaphase, anaphase and telophase) - that normally are represented by monotone decrease of corresponding cell percentage, were evidenced to be changed by radiation impact.

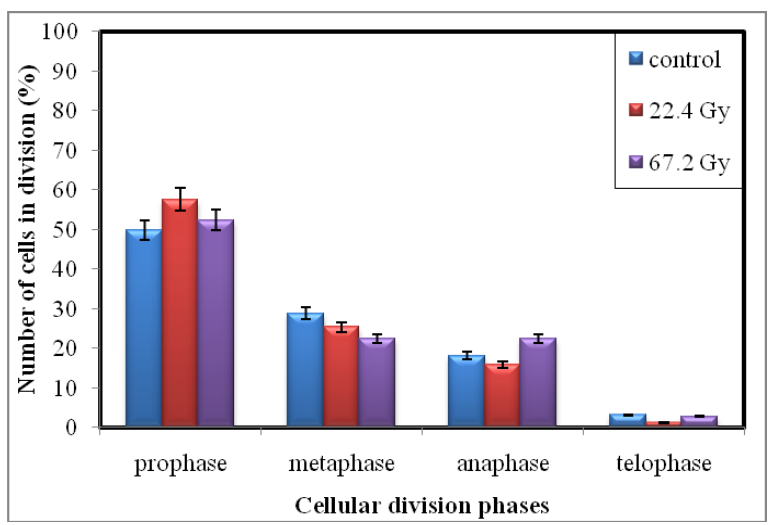

(a)

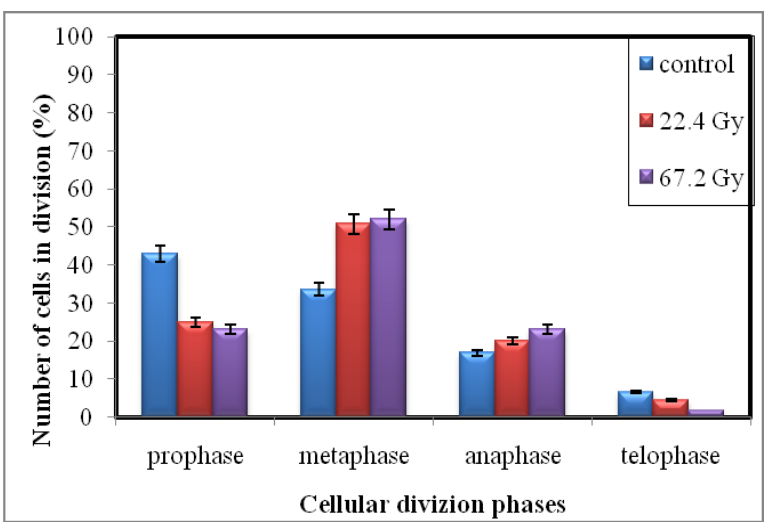

(b)

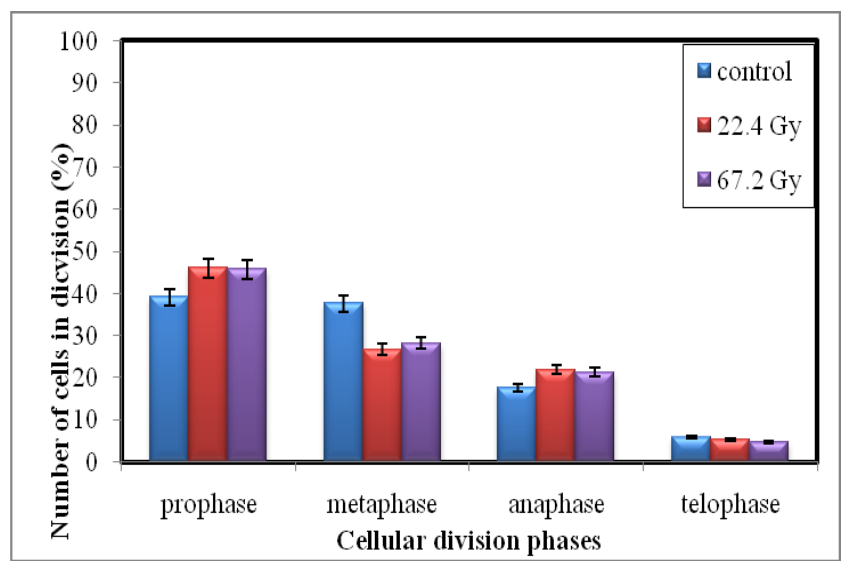

(c)

Fig. 4. Mitosis phases in a) Madalin H. vulgare b) Regal H. vulgare, c) T. aestivum

Figs. 4 (a, b, c) show an enhancing of metaphase percentage (with subsequent decrease of prophase percentage) in Madalin $H$. vulgare, but the diminution of metaphase cells together with the increasing of prophase cells in T. aestivum. No significant changes from normally frequency of phases appeared in Regal $H$. vulgare. A possible explication of abnormal mitoses induced by X-rays could be based on the general mechanisms of radiation interaction with the living cells (either directly or indirectly - by means of water radiolysis), which can result in chromosome disturbing during mitosis, meaning the appearance of chromosomal aberrations; DNA damage repair enzymes could fix some molecular damages 
but this recovery mechanism seems to depend on the plant species, being more active in Madalin barley compared to Regal barley and wheat.

\section{Conclusions}

This work demonstrated vegetal embryos sensitivity to ionizing radiations (22.4 and 67.2 Gy).The radiosensitivity was revealed at the level of both mitotic index (M.I.) and aberration percentage (A.I.); different types of chromosomal aberrations appeared to have different frequencies for the same absorbed doses in different cereal species (the cytogenetic answers depending on the plant species or plant variety for the same plant species). Considering the biotechnological issues, the radiation genetic effects in plant embryos could result not only in chromosomal aberrations but also in genetic mutations when perpetuated to the next generations so that new and possibly benefic plant features could be obtained by using ionizing radiation as biotechnological tool - which is of economic interest.

\section{References}

[1] A.T. Natarajan, Cur. sci., 89 (2005) 335;

[2] S.G. Wi, B.Y. Chung, J.S. Kim, J.H. Kim, M.H. Baek, J.W. Lee, Y.S. Kim, Micron, 38 (2007) 553;

[3] Y. Eroglu, H. E. Eroglu, A. I. Ilbas, Adv. Biol. Res., 1 (2007) 26;

[4] L. Cheng, H. Yang, B. Lin, Y. Wang, F. Zhang, Int. J. Radiat. Biol. 86 (2010) 791;

[5] H. Okamono and A. Tatara, Envir. Exper. Bot., 35 (2005) 379;

[6] N. K. Singh and H. S. Balyan, Adv. Biol. Res., 3 (2009) 215;

[7] M. Campeanu, M. Maniu and I. C. Surugiu, Genetica - metode de studiu (Genotoxic effects of ELF fields in plants), Ed. Corson, Iasi (2002). 